\title{
Metformin and Reduced Risk of Cancer in the Hong Kong Diabetes Registry: Real Effect or Immortal Time Bias?
}

\author{
Zhi-Jiang Zhang, $P h D$
}

Department of Preventive Medicine, School of Health Sciences, Wuhan University, Wuhan, China.

BACKGROUND: Whether metformin reduces cancer risk has been hotly debated. One common opinion is that the observed beneficial effects of metformin are the consequence of immortal time bias.

OBJECTIVE: To examine whether the observed beneficial effects of metformin on cancer risk are the consequence of immortal time bias.

DESIGN: Retrospective cohort study.

PARTICIPANTS: A cohort of 3485 patients who started metformin before or at enrollment, 1226 patients who initiated metformin after enrollment, and an unexposed group of 1392 patients who never used metformin.

MAIN MEASURES: Metformin users were categorized into 11 groups in terms of length of time between metformin initiation and enrollment. The percent changes in immortal person-time were calculated for each group.

RESULTS: As the groups of current metformin users ( $n=$ $3485)$ were added sequentially to the metformin group with potential immortal time bias $(n=1226)$, the proportion of immortal person-time decreased gradually by $74 \%$. As the immortal time decreased, the association between metformin and cancer risk remained statistically significant (uncorrected hazard ratio $0.54,95 \%$ confidence interval 0.42-0.69, $P<0.0001$ ).

CONCLUSION: The change in the association between metformin and cancer is small compared with the changes in the proportion of immortal time, suggesting that immortal time bias does not account for the observed beneficial effect of metformin on cancer risk. Further studies are warranted to confirm this finding in other cohort studies.

KEY WORDS: immortal time bias; metformin; cancer risk; cohort study; sensitivity analysis.

J Gen Intern Med 34(7):1154-7

DOI: $10.1007 / \mathrm{s} 11606-019-04982-\mathrm{Z}$

(C) Society of General Internal Medicine 2019

\section{INTRODUCTION}

Cumulative evidence from cohort studies suggests that metformin therapy is associated with reduced risks of cancer. ${ }^{1-4}$ However, immortal time bias exists in many previous cohort studies. $^{2-5}$ The effect estimates of metformin on cancer risk might have been affected by this bias. Some believe that the

Received August 13, 2018

Revised December 10, 2018

Accepted March 7, 2019

Published online April 25, 2019 observed reduction in cancer risk associated with metformin is the consequence of immortal time bias. ${ }^{5-8}$

Immortal time bias is a common problem in clinical epidemiology. Immortal time bias occurs when there is a period of time between enrollment and initiation of the medication. For example, in cohort studies of cancer risk comparing metformin users with non-metformin users, the cohorts may be assembled based on a specific date, e.g., enrollment date, and some of the participants start metformin therapy sometime after cohort entry. If these participants are categorized as part of the metformin group without accounting for the time span between cohort entry and metformin initiation, immortal time bias occurs (Fig. 1). The magnitude of influence from immortal time bias on the effect estimation may be minimal or large, depending on the proportion of immortal time among the total person-time in the exposed group. ${ }^{9,10}$

In this study, we used data from the Hong Kong Diabetes Registry to examine whether the observed beneficial effect of metformin on cancer risk is the consequence of immortal time bias.

\section{METHODS}

\section{Study Population}

We selected a data from previously published cohort, ${ }^{11}$ which had been selected by Suissa and colleagues as one example of immortal time bias. ${ }^{5}$ Their study was based on the Hong Kong Diabetes Registry, which was established at the Prince of Wales Hospital serving a population of over 1.2 million. ${ }^{11}$ Referred patients were enrolled once a 4-h assessment of complications and risk factors had been performed on an outpatient basis. Of 7387 patients with diabetes, 1284 were excluded from the present analysis for one of following reasons: (1) type 1 diabetes or missing data on type; (2) unknown nationality; (3) unknown history of cancer or receiving cancer treatment at enrollment; (4) missing values on any variables used in the analysis. A final cohort of 6103 patients with type 2 diabetes was formed. The primary endpoint was incident cancers after enrollment during 1996-2005.

\section{Comparison Groups}

The first metformin group was comprised of 1226 patients who started metformin after enrollment (Fig. 1). There were 


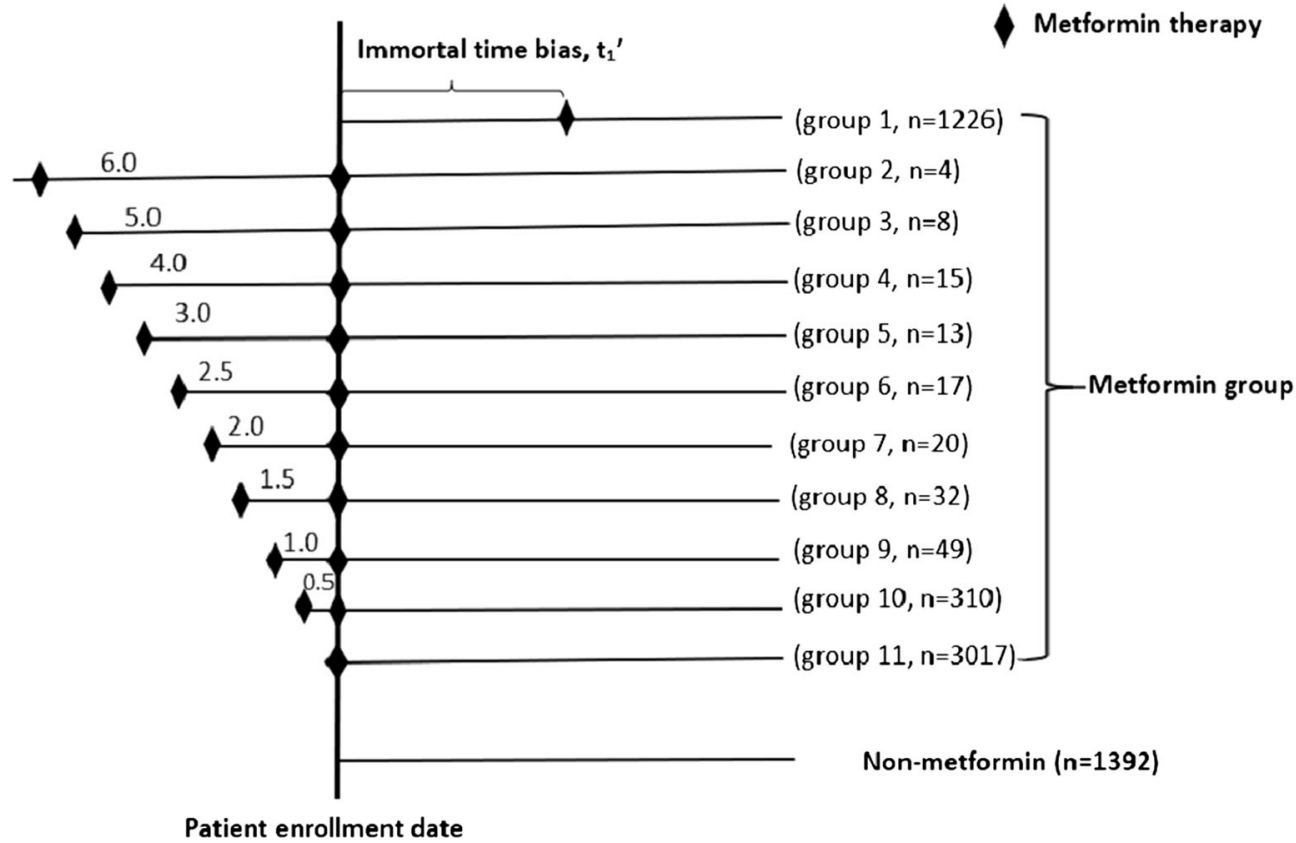

Figure 1 Study population and designs of the study. Group 1 consisted of 1226 patients who started taking metformin sometime after enrollment. The time span, $t_{1}{ }^{\prime}$, between enrollment and the date of initiating metformin is immortal time. Groups 2-11 consisted of patients who initiated metformin therapy 0-6 years before enrollment. Patients in groups 2-11 were current metformin users at enrollment. They contributed none to immortal time. Groups 2-11 were added sequentially, one at a time, to group 1 to form 10 new metformin groups with accumulative sample size. These 11 metformin groups were compared with the same unexposed group, which consisted of 1392 patients who had never used metformin either before or after enrollment.

3485 patients who started metformin some time before or at enrollment. These patients were categorized into 10 groups in terms of the time length between metformin initiation and enrollment (Fig. 1). These 10 groups were added, one at a time, to the first metformin group of 1226 patients to form 10 larger comparison groups, resulting in a series of 11 metformin groups (Table 1).

The unexposed group was comprised of 1392 patients with type 2 diabetes who never used metformin before or after enrollment (Fig. 1).

\section{Identification of Immortal Time}

The 1226 patients in the first metformin group started metformin after enrollment. The period between enrollment and the first prescription of metformin is immortal time $\left(t_{1}{ }^{\prime}\right.$, Fig. 1), because these patients had been at no risk of cancer during this time period. In contrast, the 3485 current metformin users in groups 2-11 (Table 1) and patients in the unexposed group were not subject to immortal time bias (Fig. 1).

\section{Statistical Analysis}

The formulas for assessing rates, correcting for immortal time bias, and quantifying the proportion of immortal time among the total person-time in the metformin group are provided in the appendix (appendix 1). We assessed the impact from immortal time bias on the effect size estimation of metformin by comparing the percent changes in the immortal time with the changes in the corresponding uncorrected rate ratios using univariate hazard ratios derived via Cox proportional hazards models.

\section{RESULTS}

As groups $2-11$ of current metformin users were added sequentially, one at a time, to the group of patients with potential immortal time bias ( $n=1226$, Fig. 1), the sample sizes of the newly formed metformin groups increased by $284 \%$ (from 1226 to 4711), while the proportion of immortal person-time in the newly formed metformin groups decreased gradually by $74 \%$ (Table 1).

The uncorrected hazard ratios remained relatively unchanged (from 0.37 to 0.38 ) when groups $2-10$ were added to the metformin group and the proportion of immortal time decreased by $28 \%$ (Table 1 ). When groups $2-11$ were added to the metformin group and the proportion of immortal time decreased by $74 \%$, the uncorrected hazard ratio changed from 0.37 to 0.54 (95\% confidence interval $0.42-0.69, P<0.0001$ ), corresponding to the protection rates changing from 63 to $46 \%$.

\section{DISCUSSION}

Our study examines whether the observed beneficial effect of metformin can be ascribed entirely to immortal time bias, as argued by Suissa and colleagues. ${ }^{5}$ If immortal time bias plays an important role, the magnitude of influence should be sensitive to the length of immortal time. ${ }^{9,10}$ By forming a series of comparisons, we found that the change in the association between metformin and cancer is small compared with the large changes in the proportion of immortal time, suggesting that immortal time bias does not exert a significant effect. 
Table 1 Uncorrected Hazard Ratios Corresponding to a Series of 11 Metformin Groups Formed by Sequentially Adding Each of the 10 Groups of Current Metformin Users to the Initial Group of 1226 Patients Who Started Metformin After Enrollment ${ }^{11}$

\begin{tabular}{|c|c|c|c|c|c|c|c|c|}
\hline $\begin{array}{l}\text { Metformin } \\
\text { group }\end{array}$ & $\begin{array}{l}\text { Years of } \\
\text { metformin } \\
\text { usage before } \\
\text { enrollment }\end{array}$ & $\begin{array}{l}\text { Sample } \\
\text { size in } \\
\text { each } \\
\text { group }\end{array}$ & $\begin{array}{l}\text { Immortal } \\
\text { person- } \\
\text { time }\end{array}$ & $\begin{array}{l}\text { Accumulative } \\
\text { sample sizes in } \\
\text { new metformin } \\
\text { groups }\end{array}$ & $\begin{array}{l}\text { Relative } \\
\text { decrease in } \\
\text { immortal } \\
\text { time* }\end{array}$ & $\begin{array}{l}\text { Uncorrected } \\
\text { hazard ratio }\end{array}$ & $95 \% \mathrm{CI}$ & $P$ value \\
\hline $1 \mathrm{st}$ & After enrollment & 1226 & $t_{1}^{\prime}$ & 1226 & - & 0.37 & $0.25-0.54$ & $<0.0001$ \\
\hline 2nd & 6.0 & 4 & 0 & 1230 & $0.3 \%$ & 0.37 & $0.25-0.54$ & $<0.0001$ \\
\hline 3rd & 5.0 & 8 & 0 & 1238 & $1.0 \%$ & 0.37 & $0.25-0.54$ & $<0.0001$ \\
\hline 4 th & 4.0 & 15 & 0 & 1253 & $2.0 \%$ & 0.37 & $0.25-0.54$ & $<0.0001$ \\
\hline 5 th & 3.0 & 13 & 0 & 1266 & $3.0 \%$ & 0.37 & $0.26-0.54$ & $<0.0001$ \\
\hline 6th & 2.5 & 17 & 0 & 1283 & $4.0 \%$ & 0.37 & $0.26-0.54$ & $<0.0001$ \\
\hline 7 th & 2.0 & 20 & 0 & 1303 & $6.0 \%$ & 0.37 & $0.26-0.54$ & $<0.0001$ \\
\hline 8th & 1.5 & 32 & 0 & 1335 & $8.0 \%$ & 0.37 & $0.26-0.54$ & $<0.0001$ \\
\hline 9 th & 1.0 & 49 & 0 & 1384 & $11.0 \%$ & 0.38 & $0.26-0.55$ & $<0.0001$ \\
\hline 10 th & 0.5 & 310 & 0 & 1694 & $28.0 \%$ & 0.38 & $0.27-0.54$ & $<0.0001$ \\
\hline 11 th & 0 (at enrollment) & 3017 & 0 & 4711 & $74.0 \%$ & 0.54 & $0.42-0.69$ & $<0.0001$ \\
\hline
\end{tabular}

*Relative decrease in the amount of immortal time in the newly formed metformin groups due to addition of current metformin users. Details are shown in appendix 1

${ }^{\dagger}$ The hazard ratios were derived through univariate Cox proportional hazards model without accounting for immortal time ${ }^{11}$

For example, when patients who initiated metformin at the time of enrollment (group 11) were added, the uncorrected hazard ratio changed from 0.37 to 0.54 (95\% confidence interval $0.42-0.69, P<0.0001)$, corresponding to the protection rates changing from 63 to $46 \%$. For this group, there was a $74 \%$ decrease in immortal time bias (from $T^{\prime}$ to $0.26 T^{\prime}$ ). Because most patients initiated metformin at enrollment, the sample size in this group $(n=3017)$ is much larger than that of the first metformin group $(n=1226)$ and groups $2-10$ combined $(n=468)$. This explains why the hazard ratio was more significantly changed when this group was added. Although the uncorrected hazard ratio changed from 0.37 to 0.54 , the inverse association between metformin and cancer risk remained statistically significant.

In the present study, we did not attempt to adjust for immortal time bias. Instead, we used sensitivity analysis to evaluate its potential influence. If one finds that the influence of immortal time is minimal, then Kaplan-Meier or Cox proportional hazards model (without time adjustment) is sufficient. However, when the influence of immortal time bias is large, more sophisticated statistical model is warranted, such as Cox proportional hazard model with a time-dependent covariate. $9,{ }^{10}$ One must be sure in such models that underlying assumptions are met; otherwise, the use of time-dependent covariates may introduce new bias, sometimes even larger than from immortal time bias itself. $^{12-17}$ Another approach for mitigating immortal time bias in non-randomized trials is to design the cohort appropriately. Participants who are not taking the medication of interest at enrollment could be included in the unexposed group and then censored at the point they begin taking the medication.

One implication of our study is the possibility of screening for the potential influence of immortal time bias before doing the complex statistical methods to correct for it. As our study, sensitivity analyses can be helpful in providing a quantitative assessment of the magnitude of influence due to immortal time bias. Alternatively, a Kaplan-Meir survival plot is useful as a visual examination of potential immortal time bias, with early divergence at the beginning of follow-up or crossover between the survival curves indicating potentially important immortal time bias. If no concrete evidence supporting an influential immortal time bias from either sensitivity analyses or KaplanMeier plot is found, undertaking the complex statistical calculations to account for immortal time bias is unnecessary. ${ }^{12-17}$

Our study has limitations. First, the univariate hazard ratios were not adjusted for potential confounders or prevalent user bias. However, the primary aim of this study is to explore the potential influence of immortal time bias rather than making definitive statements about the benefit of metformin in reducing cancer rates. The authors of the original report ${ }^{11}$ re-analyzed their data by excluding immortal person-time from the metformin group, and the multivariate adjusted hazard ratio was 0.57 (95\% CI 0.37-0.86), ${ }^{14}$ which did not change materially from the estimates in the original report where immortal time bias was not taken into account. ${ }^{11}$ It is reassuring that while their statistical methods were different from ours, they had similar results. Second, hazard ratios were used as an approximation of rate ratio in the present study. Although hazard ratios and rate ratios are both used for time-to-event data, hazard ratios are ratios of two instantaneous rates and required to be constant over time. Third, groups 2-11 were added to the first group of metformin sequentially from the longest to shortest duration of metformin therapy. However, this order is arbitrary.

Based on the results of sensitivity analysis, we conclude that the change in the association between metformin and cancer is small compared with the large changes in the proportion of immortal time. Immortal time bias thus likely does not fully explain the inverse association between metformin and cancer risk. Further studies are warranted to confirm this finding in other cohort studies.

Corresponding Author: Zhi-Jiang Zhang, $\mathrm{PhD}$; Department of Preventive Medicine, School of Health Sciences, Wuhan University, 115 Donghu Road, Wuhan 430071, China (e-mail: zhang22968@163. com). 
Funding Source This work was financially supported by the National Nature Science Foundation of China (grant 81641123) and the Fundamental Research Funds for the Central Universities (grant number 2042017kf0193).

\section{Compliance with Ethical Standards:}

Conflict of Interest: The author declares that he does not have a conflict of interest.

\section{REFERENCES}

1. Decensi A, Puntoni M, Goodwin P, et al. Metformin and cancer risk in diabetic patients: a systematic review and meta-analysis. Cancer Prev Res (Phila). 2010; 3: 1451-1461.

2. Zhang ZJ, Zheng ZJ, Kan $\mathbf{H}$, et al. Reduced risk of colorectal cancer with metformin therapy in patients with type 2 diabetes: a meta-analysis. Diabetes Care. 2011; 34: 2323-2328.

3. Zhang ZJ, Zheng ZJ, Shi R, Su Q, Jiang Q, Kip KE. Metformin for liver cancer prevention in patients with type 2 diabetes: a systematic review and meta-analysis. J Clin Endocrinol Metab. 2012; 97: 2347-2353.

4. Zhang ZJ, Bi Y, Li S, et al. Reduced risk of lung cancer with metformin therapy in diabetic patients: a systematic review and meta-analysis. Am J Epidemiol. 2014; 180: 11-14.

5. Suissa S, Azoulay L. Metformin and the risk of cancer: time-related biases in observational studies. Diabetes Care. 2012; 35: 2665-2673.

6. Hense HW, Geier AS. Re: "reduced risk of lung cancer with metformin therapy in diabetic patients: a systematic review and meta-analysis". Am J Epidemiol. 2014; 180: 1130-1131.

7. Schmedt $\mathbf{N}$, Azoulay L, Hense $\mathbf{S}$. Re.: "Reduced risk of lung cancer with metformin therapy in diabetic patients: a systematic review and metaanalysis". Am J Epidemiol. 2014; 180: 1216-1217.

8. Suissa S. Metformin to Treat Cancer: Misstep in Translational Research from Observational Studies. Epidemiology. 2017; 28: 455-458.

9. Suissa s. Immortal time bias in pharmaco-epidemiology. Am J Epidemiol. 2008; 167: 492-499.

10. Suissa S. Immortal time bias in observational studies of drug effects. Pharmacoepidemiol Drug Saf. 2007; 16: 241-249.

11. Yang $\mathbf{X}$, So WY, Ma RC, et al. Low HDL cholesterol, metformin use, and cancer risk in type 2 diabetes: the Hong Kong Diabetes Registry. Diabetes Care. 2011; 34: 375-380.

12. Zhang ZJ. One author replies. Am J Epidemiol. 2014; 180: 1131-1132.

13. Zhang ZJ. One author replies. Am J Epidemiol. 2014; 180: 1217-1218.

14. Yang X, Chan JC. Comment on: Suissa and Azoulay. Metformin and the risk of cancer: time-related biases in observational studies. Diabetes Care. 2012;35:2665-2673. Diabetes Care. 2013; 36: e87.

15. Yang $\mathbf{X}$, Kong AP, Luk AO, et al. Validation of methods to control for immortal time bias in a pharmacoepidemiologic analysis of reninangiotensin system inhibitors in type 2 diabetes. J Epidemiol. 2014; 24: 267-273.

16. Yang XL, Ma RC, So WY, Kong AP, Xu G, Chan JC. Addressing different biases in analysing drug use on cancer risk in diabetes in non-clinical trial settings-what, why and how? Diabetes Obes Metab. 2012; 14: 579-585.

17. Kiri VA, Pride NB, Soriano LB, Vestbo J. Inhaled corticosteroids in chronic obstructive pulmonary disease - Results from two observational designs free of immortal time bias. Am J Respir Crit Care Med. 2005; 172: 460-464

Publisher's Note Springer Nature remains neutral with regard to jurisdictional claims in published maps and institutional affiliations.

\section{APPENDIX 1 \\ Rates calculation accounting for immortal time bias}

Let $T_{1}$ denote the total person-time accumulated from the 1st metformin group of 1226 patients and $D_{1}$ denote the number of new cancer occurrences in the 1st metformin group. As the 1st metformin group started metformin after enrollment (Fig. 1), let $T$ denote the immortal person-time in the 1st metformin group.

To correct immortal time bias, we excluded the immortal person-time from the total person-time when calculating cancer rate in the 1 st metformin group. Thus, the corrected cancer rate in the 1 st metformin group is estimated by.

$\operatorname{Rate}_{I}=D_{I} /\left(T_{I}-T^{\prime}\right)$.

\section{Quantification of percent changes in immortal time}

Let $i=1,2, \ldots 10$ denote the 10 groups of current metformin users (Fig. 1). When each of the 10 group was added, one at a time, to the 1st metformin group to form a new larger exposure group, an additional amount of person-time, $T_{1 \mathrm{i}}$, and an additional amount of new cancer occurrence, $D_{1 \mathrm{i}}$, need to be incorporated in the formula of cancer rate. For example, in the newly formed 2 nd metformin group (Table 1), the total person-time at risk increased to $T_{1}-T^{\prime}+T_{11}$ and the number of new cancer increased to $D_{1}+D_{11}$. The corrected cancer rate in the newly formed 2 nd metformin group is estimated by

Rate $_{2}=\left(D_{1}+D_{l 1}\right) /\left(T_{1}-T^{\prime}+T_{11}\right)$.

Assuming the 10 groups of current metformin users were homogeneous to the 1st group of 1226 patients in terms of cancer risk, the formula of cancer rate in the newly formed 2 nd metformin group could be approximated by

$$
\begin{gathered}
\text { Rate }_{2}=\left(D_{1}+D_{I I}\right) /\left(T_{I}-T^{\prime}+T_{I I}\right) \approx D_{I} /\left(T_{I}-P_{\mathrm{i}} \times T^{\prime}\right) \\
P_{\mathrm{i}} \approx 1226 /(1226+S \mathrm{i})
\end{gathered}
$$

Here, $S_{\mathrm{i}}$ represents the sample sizes in each of the 10 groups of current users. The values of $P_{\mathrm{i}}$ represent the percent decrease in immortal time in the newly formed metformin group compared to that in the 1st metformin group. 